\title{
INTERACTIONS BETWEEN HLAWKA \\ TYPE-1 AND TYPE-2 QUANTITIES
}

\begin{abstract}
XIN LUO
Abstract. The classical Hlawka inequality possesses deep connections with zonotopes and zonoids in convex geometry, and has been related to Minkowski space. We introduce Hlawka Type-1 and Type-2 quantities, and establish a Hlawka-type relation between them, which connects a vast number of strikingly different variants of the Hlawka inequalities, such as Serre's reverse Hlawka inequality in the future cone of the Minkowski space, the Hlawka inequality for subadditive functions on abelian groups by Ressel, and the integral analogs by Takahasi et al. Besides, we announce several enhanced results, such as the Hlawka inequality for the power of measure functions. Particularly, we give a complete study of the Hlawka inequality for quadratic forms which relates to a work of Serre.
\end{abstract}

Mathematics subject classification (2020): 39B62, 47A63.

Keywords and phrases: Hlawka's inequality, quadratic form, subadditivity.

\section{REFERENCES}

[1] Wlodzimierz Fechner, Hlawka's functional inequality, Aequationes Mathematicae, 87 (2014), 71-87.

[2] H. HORNICH, Eine Ungleichung fur Vektorlangen, Math. Z. 48 (1942), 268-274.

[3] Paul Ressel, The Hornich-Hlawka inequality and Bernstein functions, J. Math. Inequal. 9 (2015), $883-888$.

[4] Denis Serre, The reverse Hlawka inequality in a Minkowski space, C. R. Math. Acad. Sci. Paris. 353 (2015), 629-633.

[5] R. Schneider And W. Weil, Zonoids and Related Topics, In: Gruber P. M., Wills J. M. (eds) Convexity and Its Applications, Birkhauser, Basel (1983).

[6] Sin-Ei Takahasi, Yasuji Takahashi And Shuhei Wada, An extension of Hlawka's inequality, Math. Inequal. Appl. 3 (2000), 63-67.

[7] Sin-Ei Takahasi, Yasuji TAKahashi And Shuhei Wada, a general Hlawka inequality and its reverse inequality, Math. Inequal. Appl. 12 (2009), 1-10.

[8] H. S. Witsenhausen, A support characterization of zonotopes, Mathematika 25 (1978), 13-16.

[9] H. S. Witsenhausen, Metric inequalities and the zonoid problem, Proc. Am. Math. Soc. 40 (1973), $517-520$ 